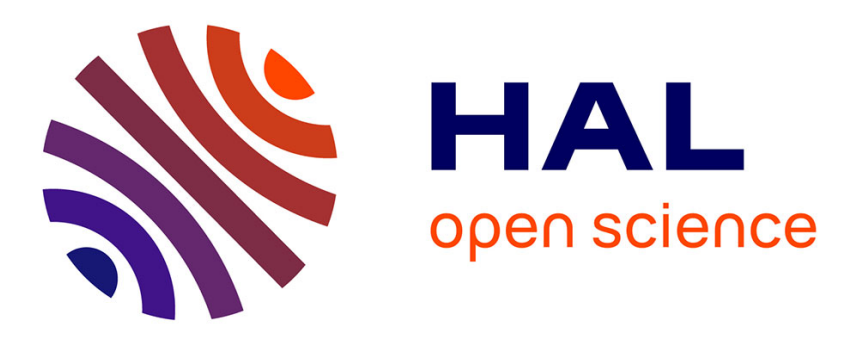

\title{
Combined longitudinal and lateral control for automated vehicle guidance
}

Rachid Attia, Rodolfo Orjuela, Michel Basset

\section{To cite this version:}

Rachid Attia, Rodolfo Orjuela, Michel Basset. Combined longitudinal and lateral control for automated vehicle guidance. Vehicle System Dynamics, 2014, 52 (2), pp.261-279. 10.1080/00423114.2013.874563 . hal-01027591

\section{HAL Id: hal-01027591 \\ https://hal.science/hal-01027591}

Submitted on 22 Jul 2014

HAL is a multi-disciplinary open access archive for the deposit and dissemination of scientific research documents, whether they are published or not. The documents may come from teaching and research institutions in France or abroad, or from public or private research centers.
L'archive ouverte pluridisciplinaire HAL, est destinée au dépôt et à la diffusion de documents scientifiques de niveau recherche, publiés ou non, émanant des établissements d'enseignement et de recherche français ou étrangers, des laboratoires publics ou privés. 


\title{
Combined Longitudinal and Lateral Control for Automated Vehicle Guidance
}

\author{
Rachid Attia*, Rodolfo Orjuela and Michel Basset \\ Modélisation Intelligence Processus Systèmes (MIPS) laboratory, EA2332 \\ Université de Haute-Alsace, 12 rue des Frères Lumière \\ F-68093 Mulhouse Cedex, France \\ (Received 00 Month 200x; final version received 00 Month 200x)
}

\begin{abstract}
This paper deals with the longitudinal and lateral control of an automotive vehicle within the framework of fully automated guidance. The automotive vehicle is a complex system characterized by highly nonlinear longitudinal and lateral coupled dynamics. Consequently, automated guidance must be simultaneously performed with longitudinal and lateral control. This work presents an automated steering strategy based on Nonlinear Model Predictive Control. A nonlinear longitudinal control strategy considering powertrain dynamics is also proposed to cope with the longitudinal speed tracking problem. Finally a simultaneous longitudinal and lateral control strategy helps to improve the combined control performance. This whole control strategy is tested through simulations showing the effectiveness of the present approach.
\end{abstract}

Keywords: vehicle guidance, nonlinear model predictive control (NMPC), longitudinal and lateral control, automated vehicle, automotive control.

\section{Introduction}

With the everyday use of automotive vehicles, the number of vehicles on roads has increased dramatically. This has led to new challenges such as passenger safety and comfort, fuel consumption optimization and the reduction of pollutant emissions. To meet these challenges, automatic control can play an important role in the development of Advanced Driver Assistance Systems (ADAS) [11]. These systems allow vehicle stabilization through Global Chassis Control (GCC) [27], driver assistance for vehicle guidance and navigation, driver warning and decision making [8], etc. Nowadays, a number of ADAS are produced by carmakers and available in automotives. More than driver assistance, ongoing research and development in the automotive field are oriented to driverless cars with the design of systems for partially/fully automated driving.

Emergence of research on fully automated driving has been largely spurred by some important international challenges and competitions, for instance, the well known DARPA Grand Challenge held in 2005 [29]. Recently, autonomous vehicle technology attracts automotive industry due to its potential applications such as automated highways, urban transportation, etc. However, fully automated driving remains a complex task which involves challenging aspects and requires skills in domains such as vision and image processing, trajectory generation path planning,

This work was supported by the Région Alsace.

*Corresponding author. Email: rachid.attia@uha.fr 
modelling and automatic control. The latter problem is of a paramount importance for vehicle guidance, i.e. steering and velocity control. As will be shown hereafter, the steering and velocity tracking problems are considered separately or in a coupled way in the literature.

Automatic vehicle steering deals with the path tracking problem. A review of steering control strategies and their implementation is presented in [28]. The study shows that in practice the performance of the steering strategies commonly implemented largely depends on the vehicle operating range and the model uncertainties. To enhance the overall performance of automated steering, more sophisticated control techniques can be used. For instance, a fuzzy control approach to deal with this problem is adopted in [19]. The fuzzy controller is compared to a classic Lyapunov controller and shows effective performance. However, stability proof and performance analysis for this fuzzy control strategy are still difficult to establish. A Neural network-based technique using genetic algorithm optimization is proposed in [23]. The major drawback of this approach is the number of driving situations needed to build a representative training data set. Besides artificial intelligence techniques, model based control methods have been also explored. Among these, model predictive control (MPC) provides interesting results; see $[2-4,7]$ and references therein. In fact, thanks to its capabilities MPC handles efficiently constrained control problems for nonlinear and uncertain systems. Recently, a Nonlinear Model Predictive Control (NMPC) approach has been proposed by the authors in a previous paper [2] where they focus on lateral control, while considering a basic longitudinal control to track the reference speed.

The speed tracking task is also relevant in fully automated driving. The Cruise Controller (CC) is widely used to ensuring vehicle speed regulation. An extension of the $\mathrm{CC}$ is the Active $\mathrm{CC}$ (ACC) which employs external information for regulation of both vehicle speed and intervehicular distance. An interesting review of the development of active cruise control systems is presented in [32]. An ACC design based on a sliding mode technique is proposed and experimentally validated in [22]. A gain-scheduling technique is also used to cope with the longitudinal control problem [26]. In fact, the vehicle operating point is modified by gear shifts and consequently, a local controller considering each operating point has been designed. It must be noted that the stability analysis of this control law is not straightforward. Nonlinear control techniques are also employed for longitudinal control design using for example a direct Lyapunov approach as proposed in [6]. In the present study, the longitudinal control problem is tackled via a similar approach based on a direct Lyapunov design. However, a robust stability design is proposed to ensure dynamic performance with respect to the longitudinal model uncertainties.

In the above studies, lateral and longitudinal control problems have been investigated in a decoupled way. In fact, numerous studies dealing with the lateral guidance of automotive vehicles are based on the assumption of a constant speed. On the other hand, those dealing with longitudinal control do not take account of the coupling with the lateral motion. However, there are strong couplings between the two dynamics at several levels: dynamic, kinematic and tyre forces (these couplings are highlighted in Section 2). Consequently, the simultaneous inclusion of longitudinal and lateral control becomes unavoidable in order to improve performance guidance in a large operating range. Nevertheless, the control design based on a complex mathematical model of the vehicle becomes a difficult task 
due to these couplings. Therefore, different control approaches have been proposed in the literature to cope with this interesting problem: for example, coupled longitudinal and lateral control based on a sliding mode technique is proposed in [17]. The idea is to calculate the desired tyre forces to obtain the steering angle by inverting the tyre model. Note that the analytical inversion of the tyre model is not possible. This makes the operation somehow complex. Recently, a solution based on the flatness control theory has been proposed in [20] and a solution based on a backstepping synthesis is proposed in [21]. The two control inputs considered are the traction torque and the steering angle. Both are calculated using a standard backstepping synthesis. The dynamics of the powertrain, the complex torque-speed relationship and the gearbox ratio are not considered in the papers cited.

In this paper, the coupled longitudinal and lateral control problem is investigated and an architecture ensuring global control is proposed. The originality of the approach lies in two contributions. The first contribution consists of the control architecture which combines the steering and the longitudinal controllers so as to ensure the simultaneous control of longitudinal and lateral motions. This approach offers the possibility to decouple the problems of path and speed tracking in order to cope with the control design for each objective separately. The second contribution consists in the use of heterogeneous criteria to update the longitudinal speed reference in order to improve the lateral stability level, thus increasing the autonomous guidance safety.

The paper is organized as follows. Section 2 presents the different models used for simulation and those used for controller synthesis. A complete 2D chassis model is presented and used for simulation purposes. From this model, a simplified model is used for controller synthesis. Section 3 and 4 respectively describe the lateral and longitudinal control design. The lateral control is based on a nonlinear model predictive synthesis and the longitudinal control is based on a direct Lyapunov approach. Section 5 presents the main contribution of this work: coupled control is detailed, the controllers presented are combined to obtain simultaneous longitudinal and lateral control. Different simulation results show the effectiveness of the proposed approach.

Notation. The following notations will be used in this paper. The subscripts $f$ and $r$ refer to front and rear. The subscripts $\{f, l / r\}$ and $\{r, l / r\}$ refer to $\{$ front,left/right $\}$ and $\{$ rear,left/right $\} .\{x, y\}$ denotes the vehicle local frame and $\{X, Y\}$ a fixed frame. $F_{x}$ and $F_{y}$ denote respectively the longitudinal and lateral forces at the vehicle centre of gravity (CoG). $F_{l}$ and $F_{c}$ are respectively the longitudinal and lateral tyre forces.

\section{Automotive Vehicle Modelling}

Mathematical models are of great importance in the analysis and control of automotive vehicle dynamics. Several mathematical models are available in the literature with different levels of complexity and accuracy according to the physical phenomena captured [15, 25]. Here, the motion of the vehicle is investigated in the yaw plane mainly describing the longitudinal and lateral vehicle motion. In the description of the vehicle motion, different longitudinal and lateral dynamic couplings must be considered:

- Dynamic and kinematic couplings are due to the motion in the yaw plane caused 
by wheels steering.

- The interaction between tyre and road is at the origin of another important coupling. In fact, the maximal available tyre-road friction is distributed between lateral and longitudinal tyre forces. This distribution is governed by the well known friction ellipse [15].

- The longitudinal and lateral accelerations cause a load transfer between the front and rear axles as well as the right and left wheels. These load transfers affect the vertical dynamics as well as the lateral and longitudinal ones due to the modification in the normal tyre forces.

Here, two mathematical models of different complexity degrees are used to obtain a trade-off between complexity and accuracy. In fact, a model is used for validation issues through numerical simulations and a second one for control design. The validation model is a $2 \mathrm{D}$ chassis model capturing the chassis longitudinal and lateral as well as the tyre dynamic couplings. This model also captures the powertrain dynamics, i.e. the engine map and the evolution of the gearbox. The resulting model provides a good accuracy level but remains too complex for controller synthesis. This complexity results from the nonlinear engine map, the discrete evolution of the gearbox ratio, the coupling characterizing the vehicle dynamics and the tyreroad behaviour. Therefore, a nonlinear bicycle model is used for lateral control and a one wheel vehicle model for longitudinal control design.

\subsection{Validation Model}

The validation model is composed of a $2 \mathrm{D}$ model of the chassis, Burckhardt's tyre model and a powertrain model presented below.

\subsubsection{D Chassis model}

Figure 1 shows a 2D model of the vehicle motion with the main forces acting on the vehicle. Let $x$ and $y$ respectively be the longitudinal and lateral directions in the vehicle frame, $X$ and $Y$ the longitudinal and lateral directions in the absolute frame, $\psi$ the yaw angle in the $\{x, y\}$ frame and $\Psi$ the heading angle in the $\{X, Y\}$ frame. The Euler-Newton formalism allows the expression of the chassis dynamics in the vehicle frame as follows:

$$
\begin{aligned}
m \ddot{x}= & m \dot{y} \dot{\psi}+F_{x_{f, l}}+F_{x_{f, r}}+F_{x_{r, l}}+F_{x_{r, r}}, \\
m \ddot{y}= & -m \dot{x} \dot{\psi}+F_{y_{f, l}}+F_{y_{f, r}}+F_{y_{r, l}}+F_{y_{r, r}}, \\
I \ddot{\psi}= & a\left(F_{y_{f, l}}+F_{y_{f, r}}\right)-b\left(F_{y_{r, l}}+F_{y_{r, r}}\right) \\
& +c\left(-F_{x_{f, l}}+F_{x_{f, r}}-F_{x_{r, l}}+F_{x_{r, r}}\right),
\end{aligned}
$$

where $m$ and $I$ are respectively the vehicle mass and the moment of inertia, $a$ and $b$ are the front and rear CoG-distances, $c$ the track-width, $F_{x_{., .}}$and $F_{y_{1 .,}}$ are the forces respectively in the $x$ and $y$ directions.

The vehicle coordinates in the fixed frame are calculated using the kinematic model given by: 


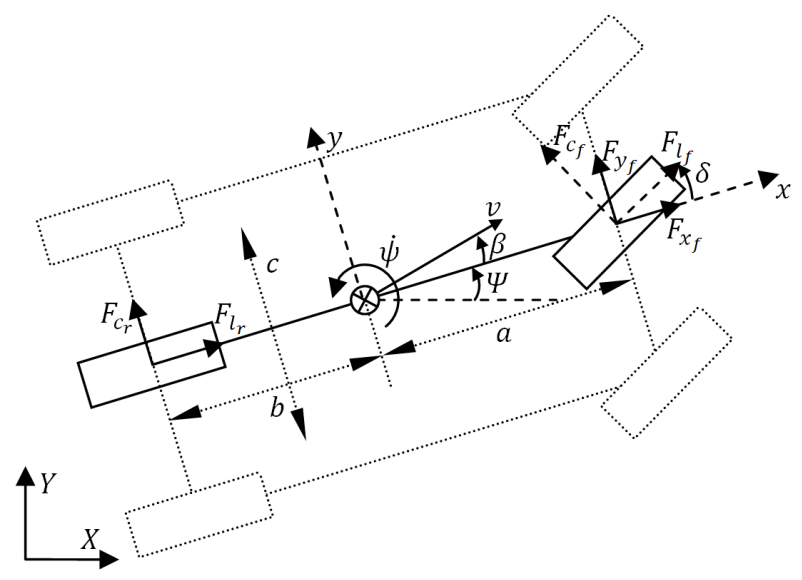

Figure 1. 2DOF model of the vehicle.

$$
\begin{aligned}
\dot{X} & =\dot{x} \cos \Psi-\dot{y} \sin \Psi, \\
\dot{Y} & =\dot{x} \sin \Psi+\dot{y} \cos \Psi, \\
\dot{\Psi} & =\dot{\psi} .
\end{aligned}
$$

The forces $F_{x_{f, r} / l, r}$ and $F_{y_{f, r} / l, r}$ acting on the CoG of the vehicle are related to the tyre forces and the front steering angle $\delta$ as follows:

$$
\begin{aligned}
& F_{x_{f, l / r}}=F_{l_{f, l / r}} \cos \delta-F_{c_{f, l / r}} \sin \delta, \\
& F_{y_{f, l / r}}=F_{l_{f, l / r}} \sin \delta+F_{c_{f, l / r}} \cos \delta, \\
& F_{x_{r, l / r}}=F_{l_{r, l / r}}, \\
& F_{y_{r, l / r}}=F_{c_{r, l / r}} .
\end{aligned}
$$

For the sake of simplicity, the steering angles of the left and right front wheels are supposed to be equal.

The forces at the tyre ground contact point result from the wheels dynamics given by the following equations:

$$
\begin{aligned}
I_{w} \dot{\omega}_{f, l} & =-F_{l_{f, l}} R+T_{t_{f, l}}-B_{d} \omega_{f, l}, \\
I_{w} \dot{\omega}_{f, r} & =-F_{l_{f, r}} R+T_{t_{f, r}}-B_{d} \omega_{f, r}, \\
I_{w} \dot{\omega}_{r, l} & =-F_{l_{r, l}} R+T_{t_{r, l}}, \\
I_{w} \dot{\omega}_{r, r} & =-F_{l_{r, r}} R+T_{t_{r, r}}
\end{aligned}
$$

where $T_{t . . .}$ is the total torque applied on each wheel (traction and brake torques), $\omega_{\text {... }}$ the wheel rotational speed, $I_{w}$ the moment of inertia of the wheel, $R$ the wheel radius and $B_{d}$ a damping coefficient.

\subsubsection{Tyre model}

The main external forces acting on the vehicle result from the tyre-road interaction which largely affects the longitudinal dynamics, particularly in important acceleration phases as pointed out in [6]. Therefore, the use of an accurate tyre force model is of the utmost importance to obtain a realistic vehicle motion 
dynamics and to capture nonlinear behaviour in hard lateral manoeuvres. Among the different existing tyre models, Burckhardt's model [15] has been chosen here. Indeed, this model helps to consider both vehicle speed and vertical forces through a low number of parameters, compared to Pacejka's model for example. Furthermore, it takes account of the couplings of longitudinal and lateral tyre forces through the friction circle.

The tyre forces which describe Burckhardt's model are given by [15]:

$$
\begin{aligned}
& F_{l}=\mu_{\text {res }}\left(\frac{s_{L}}{s_{\text {Res }}} \cos \alpha-k_{S} \frac{s_{S}}{s_{\text {Res }}} \sin \alpha\right) F_{z}, \\
& F_{c}=-\mu_{\text {res }}\left(k_{S} \frac{s_{S}}{s_{\text {Res }}} \cos \alpha+\frac{s_{L}}{s_{\text {Res }}} \sin \alpha\right) F_{z},
\end{aligned}
$$

where $F_{l}$ and $F_{c}$ respectively are the longitudinal and lateral tyre forces, $\mu_{r e s}$ the friction coefficient, $\alpha$ the wheel side-slip angle, $F_{z}$ the vertical load and $k_{S}$ a factor varying in the interval $[0.9,0.95]$. The longitudinal and lateral sliding $s_{L}$ and $s_{S}$ are given by:

$$
\begin{gathered}
s_{L}=\frac{v_{R} \cos \alpha-v_{w}}{\max \left(v_{w}, v_{R} \cos \alpha\right)}, \\
s_{S}=\left\{\begin{array}{ll}
\left(1+s_{L}\right) \tan \alpha & \text { if } s_{L}<0 \\
\tan \alpha & \text { if } s_{L}>0
\end{array},\right.
\end{gathered}
$$

where $v_{w}$ the wheel ground point velocity and $v_{R}$ is the rotational equivalent wheel velocity given by:

$$
v_{R}=R \omega,
$$

where $R$ and $\omega$ are defined in (4).

The resulting sliding $s_{\text {Res }}$ is calculated as follows:

$$
s_{\text {Res }}=\sqrt{s_{L}^{2}+s_{S}^{2}} .
$$

The friction coefficient $\mu_{\text {Res }}$ is calculated using Burckhardt's model [15]:

$$
\mu_{\text {Res }}\left(s_{\text {Res }}\right)=c_{1}\left(1-\exp \left(-c_{2} s_{\text {Res }}\right)\right)-c_{3} s_{\text {Res }},
$$

where parameters $c_{1}, c_{2}$ and $c_{3}$ are related to the road conditions i.e. cohesion coefficient characteristics for different road surfaces.

\subsubsection{Powertrain model}

Modelling powertrain is a difficult task due to the complex mechanical behaviour at the different links. Moreover, it is difficult to obtain representative identified parameters without a specified bench. So, a black box model is used in the validation model of the vehicle. The powertrain model considered for validation is available in MATLAB ${ }^{\circledR} /$ Simulink [2]. Figure 2 shows the structure of the model considered.

The model takes account of the engine map, the gearbox ratio changes, the dynamics and losses at mechanical links. This model is only used for validation through 
simulations; a less complex longitudinal model is presented in Section 2.2 for controller synthesis.

\subsubsection{Validation model}

Figure 2 summarizes the structure of the validation model with the focus on the inputs-outputs of each block. The figure shows the physical control inputs of the model -the steering wheel, the throttle and the brake - and shows how these control inputs act on the chassis dynamics. Note that the steering angle $\delta$ is involved in both tyre and force models. Also note the coupling of wheels and chassis models through the computation of the sliding. The whole vehicle model is used in simulation for the validation of the control laws designed.

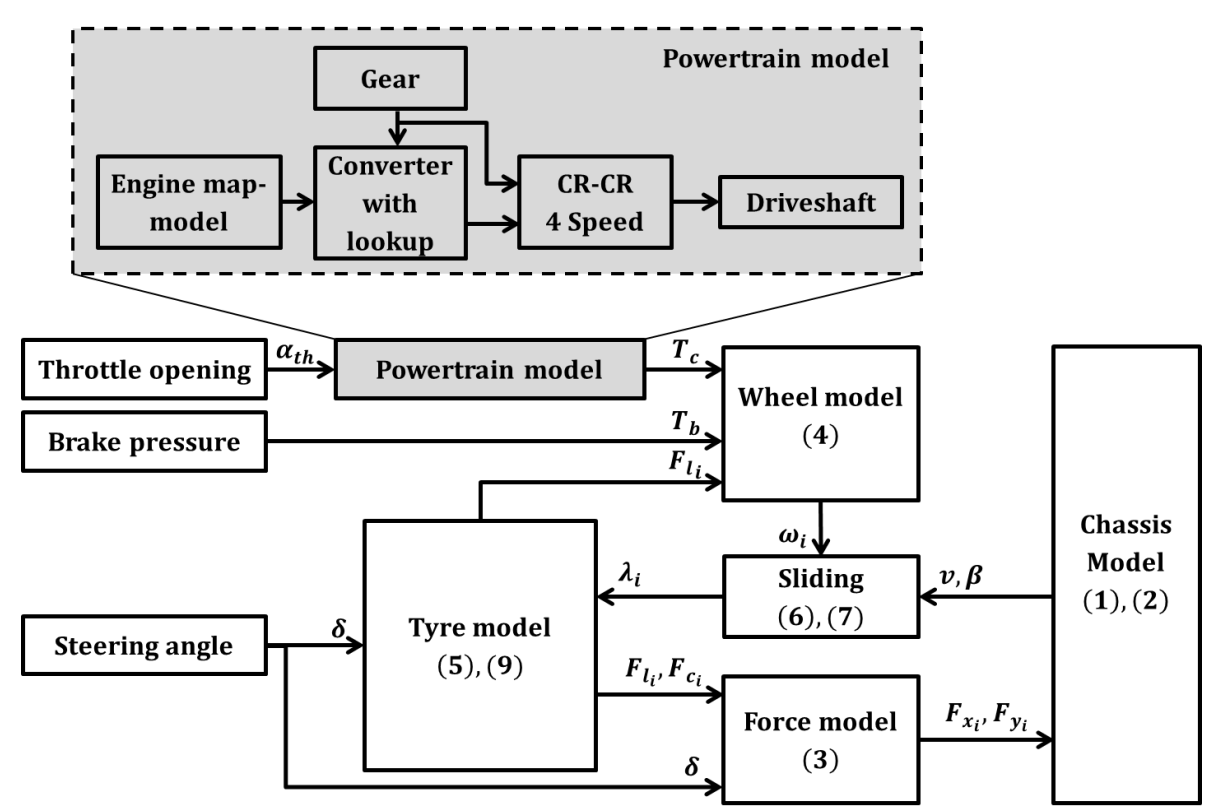

Figure 2. Structure of the vehicle validation model.

\section{2. $\quad$ Models for Control Synthesis}

In order to simplify the design of controllers, less complex but tractable models are used. The nonlinear bicycle model helps to describe the lateral motion dynamics of the vehicle. The bicycle model is obtained from the $2 \mathrm{D}$ model presented in the previous section. The longitudinal motion dynamics is described by a nonlinear model based on a one wheel vehicle representation. Both models will be described hereafter.

\subsubsection{Nonlinear bicycle model}

Under some assumptions, the nonlinear bicycle model is able to describe the main lateral dynamics needed for controller synthesis. It is supposed that the vehicle is symmetrical about the longitudinal plane, i.e. the left and right sides are identical. By collapsing the right and the left wheels for each axle as illustrated in Figure 1, 
the forces acting at the front and rear of the vehicle become:

$$
\begin{gathered}
F_{x_{f}}=F_{x_{f, l}}+F_{x_{f, r}}, \\
F_{x_{r}}=F_{x_{r, l}}+F_{x_{r, r},}, \\
F_{y_{f}}=F_{y_{f, l}}+F_{y_{f, r}}, \\
F_{y_{r}}=F_{y_{r, l}}+F_{y_{r, r}} .
\end{gathered}
$$

Using equations (1)-(4), the mathematical model of the bicycle dynamics is finally given by:

$$
\begin{aligned}
m \ddot{x} & =m \dot{y} \dot{\psi}+F_{x_{f}}+F_{x_{r}}+F_{r}, \\
m \ddot{y} & =-m \dot{x} \dot{\psi}+F_{y_{f}}+F_{y_{r}}, \\
I \ddot{\psi} & =a F_{y_{f}}-b F_{y_{r}}, \\
I_{w_{f}} \dot{\omega}_{f} & =-F_{l_{f}} R+T_{t_{f}}-B_{d} \omega_{f}, \\
I_{w_{r}} \dot{\omega}_{r} & =-F_{l_{r}} R+T_{t_{r}} .
\end{aligned}
$$

\subsubsection{Longitudinal synthesis model}

The longitudinal model considered here for controller synthesis is based on a one wheel vehicle model. The sum of the longitudinal forces acting on the vehicle CoG is given by:

$$
m \dot{v}=F_{p}-F_{r}
$$

where $v$ is the vehicle speed, $F_{p}$ is the propelling force and $F_{r}$ the sum of resisting forces. The propelling force $F_{p}$ is the controlled input resulting from brake and throttle actions. The resisting force $F_{r}$ is given by:

$$
F_{r}=F_{a}+F_{g}+F_{r r}
$$

where:

- $F_{a}=\frac{1}{2} \rho C_{d} v^{2}$ is the aerodynamic force with $\rho$ the air density and $C_{d}$ the drag coefficient.

- $F_{g}=m g \sin \theta$ is the gravitational force with $\theta$ the road slope and $g$ the gravitational acceleration.

- $F_{r r}=C_{r} m g \cos \theta$ is the rolling resistance force with $C_{r}$ the rolling resistance coefficient.

The equation (4) describing the wheel dynamics has been slightly modified so as to distinguish the brake torque $T_{b}$ and the traction torque $T_{c}$ as follows:

$$
I_{w} \dot{\omega}=-F_{l} R+T_{c}-T_{b}
$$

For longitudinal controller synthesis, the following simplifying assumptions are considered: 
- For a non-slip rolling then the following relationships hold:

$$
\begin{aligned}
v & =R \omega, \\
F_{p} & =F_{l} .
\end{aligned}
$$

- Losses between the engine and the final driveshaft are neglected, so:

$$
\begin{gathered}
\omega=R_{g} \omega_{e}, \\
T_{e}=R_{g} T_{c},
\end{gathered}
$$

where $R_{g}$ is the gearbox ratio, $\omega_{e}$ the engine speed and $T_{e}$ the engine torque.

- The throttle opening ratio $\alpha_{t h}$ is proportional to the engine power. Note that the throttle opening is the effective control input.

From (16b) and (15), the following equation is obtained:

$$
F_{p}=\frac{1}{R}\left(T_{p}-T_{b}-I_{w} \dot{\omega}\right)
$$

and the longitudinal dynamics defined by (15) becomes:

$$
\left(m+\frac{I_{w}}{R^{2}}\right) \dot{v}=\frac{T_{p}-T_{b}}{R}-F_{r}
$$

using (16a) and (18) into (13). Finally, the longitudinal dynamics is given by:

$$
\frac{\left(m R^{2}+I_{w}\right) R_{g}}{R} \dot{v}=T_{e}-R_{g} T_{b}-R_{g} R F_{r}
$$

It must be emphasized that the controlled input $T_{e}$ depends on the throttle opening ratio $\alpha_{t h}$ as well as the engine speed $\omega_{e}$. The static relationship between $T_{e}$ and $\omega_{e}$ can be obtained by the following polynomial expression [12]:

$$
P=a_{1}+a_{2} \omega_{e}+a_{3} \omega_{e}^{2}
$$

where $P=T_{e} \omega_{e}$ is the engine power and $a_{1}, a_{2}$ and $a_{3}$ are empirical model parameters. For a given engine speed, the maximum available torque is:

$$
T_{\text {emax }}=\frac{P_{\max }}{\omega_{e}} .
$$

\section{Lateral Guidance}

The lateral control problem is complex due to the longitudinal and lateral coupled dynamics as well as the tyre behaviour. These phenomena are well captured in a simplified way by the synthesis model presented in Section 2.2. As this model remains nonlinear, Nonlinear Model Predictive Control (NMPC) is adopted. Indeed, NMPC is an optimization based method for feedback control of nonlinear systems (for details see [10]). The next section presents controller design and the results obtained with this approach. 


\subsection{Nonlinear Lateral Control Design}

The basis of the MPC is to use a prediction model to calculate the future states of a dynamic system on a fixed finite time-horizon $N_{p}$ named prediction horizon. At a given time $k$, from the predictions at the instants $\left\{k, k+1, \ldots, k+N_{p}\right\}$ a cost function is minimized under given constraints [31]. At time $k+1$, the same problem is solved on the sliding horizon $\left\{k+1, k+2, \ldots, k+N_{p}+1\right\}$ and so on. Generally, the cost function to be minimized takes the following form [31]:

$$
J=\sum_{n=1}^{N p}\left\|h(k+n)-h_{r e f}(k+n)\right\|_{Q}+\sum_{n=0}^{N c-1}\|u(k+n)\|_{R},
$$

where $h$ and $h_{r e f}$ are respectively the predicted and the reference outputs, $N_{c}$ is the control horizon defining the optimization problem dimension. The weighting matrices $Q$ and $R$ respectively represent the weights regarding the tracking errors and the control input energy. Parameters $N_{p}, N_{c}, Q$ and $R$ determine the performance of the MPC feedback control.

The adopted NMPC is a discrete-time strategy and the synthesis model should be discretized. For this purpose, an approximation of the time-derivative using Euler's method is considered as follows:

$$
\xi(k+1)=\xi(k)+T_{s} f(\xi(k), u(k)),
$$

where $f(\xi, u)$ is the state-space representation of the vehicle model, $\xi=\left[\begin{array}{lllllll}\dot{x} & \dot{y} & \dot{\psi} & \omega_{f} & \omega_{r} & X & Y\end{array}\right]^{T}$ the state-space vector and $u=\delta$ the steering angle i.e. the control input. The sample time considered here is $T_{s}=10 \mathrm{~ms}$ which is a standard sampling in the automotive technology.

Note that a path tracking formulation of the lateral control problem is proposed here. The lateral position of the vehicle is defined by the coordinates $\{X, Y\}$ and the heading angle $\Psi$; they define the reference signal $h_{\text {ref }}=\left[\begin{array}{lll}X & Y & \Psi\end{array}\right]^{T}$ in (23). Thanks to this geometric reference trajectory, variable longitudinal speed during lateral manoeuvres can by handled conversely to other studies [16], [33] and [3]. The geometric reference trajectory $h_{r e f}$ can be computed at each sample-time according to the vehicle speed. The NMPC problem is then formulated as follows [2]:

$$
\begin{array}{ll} 
& \arg \min _{\Delta U} J(\xi(k), \Delta U), \\
\text { s.t } & \xi(k+1)=f_{d}(\xi(k), \Delta u(k)), \\
& h(k)=[X(k) Y(k) \Psi(k)]^{T}, \\
& u_{\min } \leq u(k) \leq u_{\max }, \\
& \Delta u_{\min } \leq \Delta u(k) \leq \Delta u_{\max }, \\
& u(k)=u(k-1)+\Delta u(k), \\
& \Delta u(k)=0 \text { for } k=N_{c}, \ldots, N_{p},
\end{array}
$$

where $f_{d}(.,$.$) is the discrete state-space representation given by (24), \Delta U=$ $\left[\Delta u(k), \ldots, \Delta u\left(k+N_{c}-1\right)\right]$ is the optimization vector, $u_{\min }$ and $u_{\max }$ are the lower and upper limits of the control input $u, \Delta u_{\min }$ and $\Delta u_{\max }$ are the lower and upper limits of the control input variation. The closed-loop control law is calculated from 
the solution $\Delta U^{*}$ of the problem (25) as follows:

$$
u(k)=u(k-1)+\Delta U^{*}(1),
$$

and at time $k+1$, the same operation is repeated on the time horizon $\left[k+1, k+N_{p}+1\right]$.

It can be noted that the constraints can be easily included in the NMPC formulation (25). Remark that several training parameters must be chosen to ensure stability and feasibility of the problem. The choice of the cost function, the weighting matrices and the prediction (and control) horizons are of great importance. Indeed, the choice of a quadratic function is motivated by the use of the quadratic error metric which is classically used in optimal control design. An adequate choice of the weighting matrices allows a trade-off between the tracking error value and the control signal energy. A high energy control signal is reflected by a high excitation of the actuator which may be undesirable in practice. Note that the stability of the NMPC scheme strongly depends on the constraints (25d) and (25e) to prevent from steering saturation. The weighting matrices, the prediction and control horizons as well as the limits of the constraints are tuned, considering the knowledge of the system and the desired performance [10,31].

\subsection{Simulation Results}

The reference trajectory to be tracked by the vehicle is obtained from the reference generation level (see Figure 7). For further details on the reference generation aspects see [1].

The proposed control strategy is tested through simulations. The nonlinear optimization problem (25) is solved using an interior-point algorithm. Two tests are presented below to evaluate the proposed control strategy.

Test 1. Lateral guidance using real-world track data: this test consists in following a track obtained from real-world GIS/Cartography [5]. Path-following is performed at a constant speed of $15 \mathrm{~m} / \mathrm{s}(54 \mathrm{~km} / \mathrm{h})$. The results of this test are shown in Figure 3. NMPC offers good tracking performance as the maximum lateral error does not exceed $5 \mathrm{~cm}$.

Test 2. Double lane-change manoeuvre: this test consists in a double lane-change manoeuvre (obstacle avoidance manoeuvre). The objective of this test is to evaluate the behaviour of the proposed control strategy in critical situations. The reference trajectory considered for this manoeuvre has been presented in [7]. It can be seen in Figure 4 that NMPC offers good tracking performance even for this kind of critical manoeuvres. In fact, the tracking errors (lateral position and heading angle) and the sideslip angle remain admissible.

\section{Longitudinal Control}

This section is devoted to the longitudinal control design. The speed tracking problem is tackled using a direct Lypunov approach; a specific robust stability analysis is provided to deal with model uncertainties. Furthermore, the management of the gear shifts and the switching between throttle and brake is discussed. Finally, the control design is tested and validated through numerical simulations. 


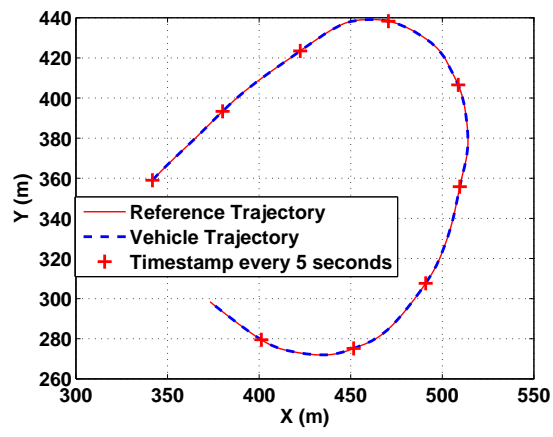

(a) Path-following

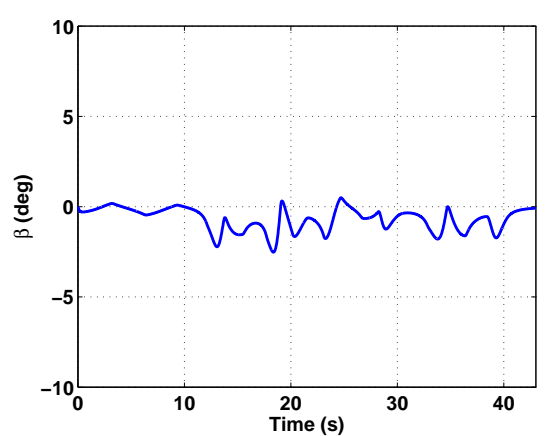

(c) Sideslip angle

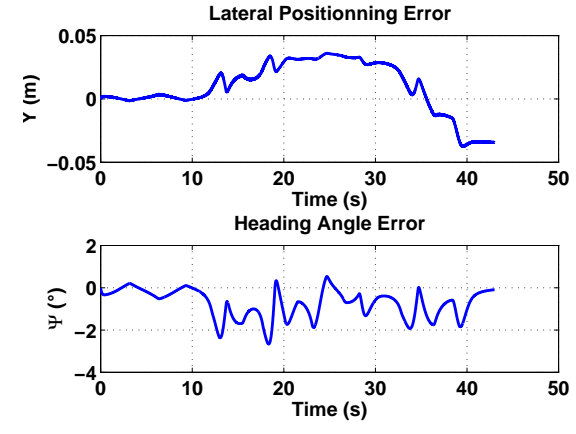

(b) Tracking errors

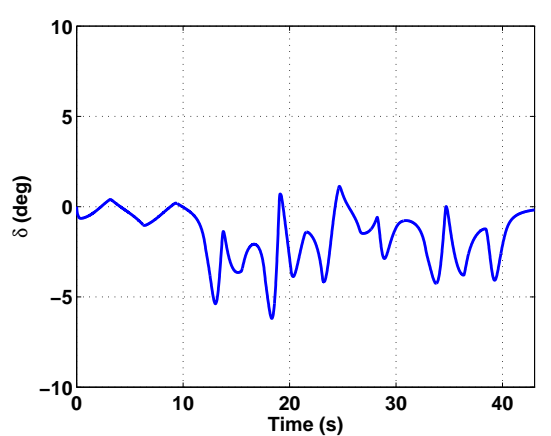

(d) Controlled steering input

Figure 3. Lateral guidance test at constant speed $15 \mathrm{~m} / \mathrm{s}$.

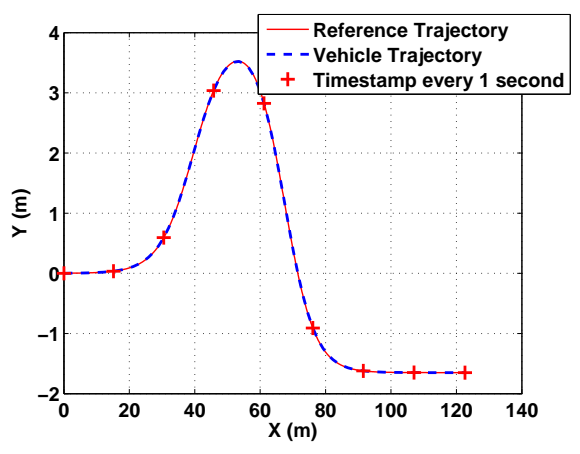

(a) Lateral position

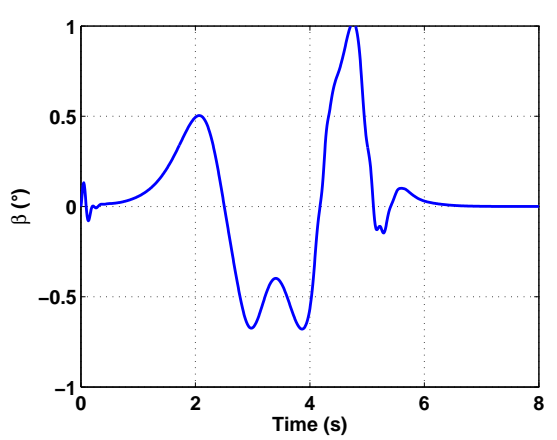

(c) Sideslip angle

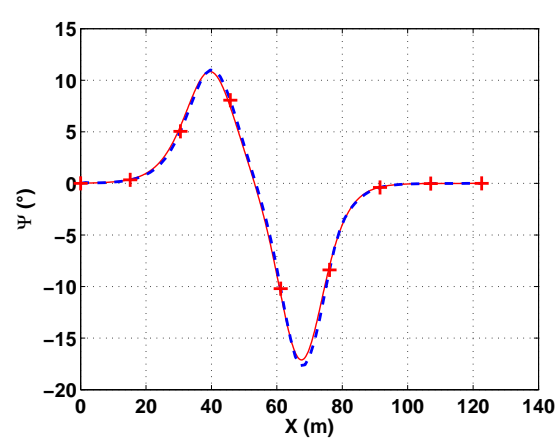

(b) Heading angle

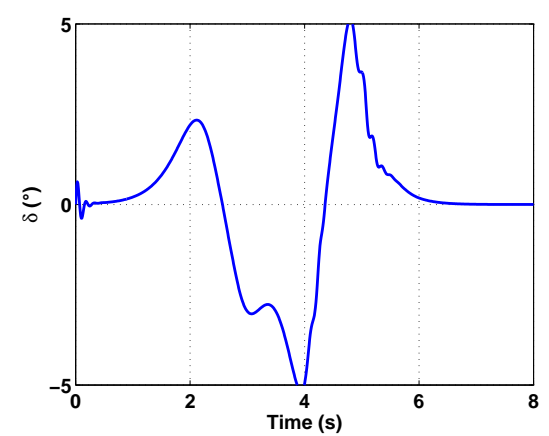

(d) Controlled steering input

Figure 4. Obstacle avoidance manoeuvre with entry speed $15 \mathrm{~m} / \mathrm{s}$. 


\subsection{Management of Longitudinal Control Actuators}

As mentioned above, the control inputs for longitudinal control are the throttle opening ratio $\alpha_{t h}$, the brake torque $T_{b}$ and the gear ratio $R_{g}$. A management policy should be defined to handle the exclusivity working between throttle and brake. To obtain realistic driving scenarios, a gear shift policy must also be determined .

The switching between throttle and brake is defined using a policy taking account of the throttle opening value given by the proposed longitudinal controller and the speed tracking error. The brake is activated if the throttle is inactive $\left(\alpha_{t h}=0\right)$ and the vehicle speed is greater than the reference speed. To avoid the chattering phenomenon, a dead-zone is introduced.

The automatic gear shift management, i.e. determining the adequate $R_{g}$ at each time-instant, is a complex optimization problem [13] and will not be dealt with here. However, several studies show that the optimal engine operating point for small road gradients is reached at around $2750 \mathrm{rpm}$ [18]. Based on this observation, a simple gear shift policy is adopted resulting in an automatic gearbox-like system modelled by the statechart shown in Figure 5.

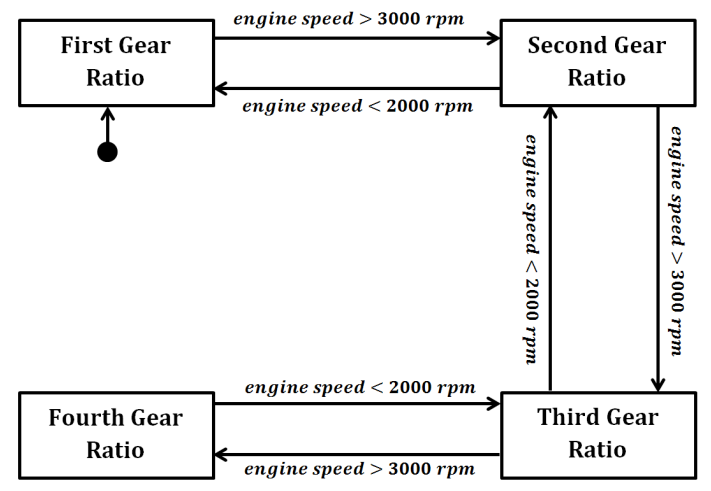

Figure 5. Gear shift policy.

\subsection{Nonlinear Longitudinal Control Design}

The proposed controller is synthesized using a Lyapunov-based approach. Consider the speed tracking error given by:

$$
e=v_{r e f}-v
$$

where $v$ and $v_{\text {ref }}$ are the actual and reference speeds. The time evolution of this error is:

$$
\dot{e}=\dot{v}_{r e f}-\dot{v}
$$

which can be rewritten as:

$$
\dot{e}=\dot{v}_{r e f}-\frac{1}{M_{t}}\left(T_{e}-R_{g}\left(T_{b}+R F_{r}\right)\right)
$$

where $M_{t}=\left(\left(m R^{2}+I_{w}\right) R_{g}\right) / R$, using the expression of $\dot{v}$ given by the the nonlinear longitudinal model (20). 
In order to ensure the convergence towards zero of the tracking error (27), a Lyapunov approach is employed; therefore, the following definite positive Lyapunovcandidate function is considered:

$$
V=\frac{1}{2} e^{2}
$$

and its time-derivative:

$$
\dot{V}=e \dot{e}
$$

The exponential convergence towards zero of the tracking error is ensured if the following condition is verified

$$
\dot{V}=-k V
$$

where $k>0$ can be considered as a decay rate.

Replacing (29) into (31) gives:

$$
\dot{V}=e\left(\dot{v}_{r e f}-\frac{1}{M_{t}}\left(T_{e}-R_{g}\left(T_{b}+R F_{r}\right)\right)\right)
$$

and considering (33) and $T_{b}=0$ (when throttle is active the brake is inactive), the stability condition (32) suggests the following nonlinear control law:

$$
T_{e}^{*}=M_{t}\left(k e+\dot{v}_{r e f}\right)+R_{g} R F_{r}, \quad k>0
$$

Note that the controlled input applied to the vehicle is the throttle opening $\alpha_{t h}$. The latter is obtained from the required engine torque $T_{e}^{*}$ provided by the control law (34) using:

$$
\alpha_{t h}=\frac{T_{e}^{*} \omega_{e}}{P_{\max }}
$$

where $\omega_{e}$ is the actual engine speed and $P_{\max }$ the maximum engine power.

\subsection{Robust Stability Analysis}

The stability condition (32) is met for the control torque $T_{e}^{*}$ when the model and the physical system match. To overcome this strong assumption, the control law (34) is robustified by considering physical parameter uncertainties in the controller synthesis.

A Robustification term $\rho$ is added into the nonlinear control law (34) as follows:

$$
\hat{T}_{e}=\hat{M}_{t}\left(k e+\dot{v}_{r e f}\right)+R_{g} \hat{R} \hat{F}_{r}+\rho
$$

where the hat designates the parameters nominal values. The robustification term $\rho$ must be determined to ensure the robust convergence of the tracking error. Considering the control law (36), the tracking error dynamics (28) becomes:

$$
\dot{e}=-\frac{\hat{M}_{t}}{M_{t}} k e+\frac{1}{M_{t}}\left(\left(M_{t}-\hat{M}_{t}\right) \dot{v}_{r e f}+\left(R g\left(M_{r}-\hat{M}_{r}\right)-\rho\right)\right)
$$


where $M_{r}=R F_{r}$ and $\hat{M}_{r}=\hat{R} \hat{F}_{r}$. Using the same Lyapunov function (30), its time derivative (31) and the tracking error dynamics (37), the following equation is obtained:

$$
\dot{V}=-\frac{\hat{M}_{t}}{M_{t}} k e^{2}+\frac{1}{M_{t}}\left(\tilde{M}_{t} \dot{v}_{r e f}+R_{g} \tilde{M}_{r}-\rho\right) e
$$

where $\tilde{M}_{t}=M_{t}-\hat{M}_{t}$ and $\tilde{M}_{r}=M_{r}-\hat{M}_{r}$.

Using (38) and the exponential stability condition (32), the following robustification term is proposed:

$$
\rho=\Delta \operatorname{sign}(e)
$$

where $\Delta>\tilde{M}_{t} \dot{v}_{r e f}+R_{g} \tilde{M}_{r}$. The robustified control law is finally given by:

$$
\hat{T}_{e}=\hat{M}_{t}\left(k e+\dot{v}_{r e f}\right)+R_{g} \hat{M}_{r}-\left(\tilde{M}_{t}^{\max } \dot{v}_{r e f}+R_{g} \tilde{M}_{r}^{\max }\right) \operatorname{sign}(e), \quad k>0
$$

where $\tilde{M}_{t}^{\text {max }}$ is the maximum uncertainty on parameter $M_{t}$ and $\tilde{M}_{r}^{\max }$ the maximum uncertainty on the total resisting moment $M_{r}$. From a practical point of view, the sign function is replaced by a saturation function to avoid the chattering phenomenon.

\subsection{Simulation Results}

This test consists in tracking different reference speeds. It is assumed that the reference speed is a continuously differentiable signal available in real-time. The evolution of the vehicle speed to track the reference in acceleration and deceleration phases is shown in Figure 6(a). Notice on Figure 6(c) the gear shifts at time instants $\{20.2 s, 30 s, 44.8 s, 61.4 s, 71.4 s, 81.1 s\}$. In fact, in acceleration phases, the increasing reference speed needs more propelling torque, then the engine speed increases and reaches the maximal value when a gear shift becomes necessary.

The evolution of the engine speed is shown in Figure 6(b). It can be seen that the engine speed remains in the neighbourhood of the optimal operating range considered thanks to the gear shift policy.

Figure 6(d) shows the throttle and brake activation in acceleration and deceleration phases. The switching between throttle and brake is effective in deceleration phases. Note that at the origin time $(t=0)$ a slight brake torque is applied to track the reference speed. In fact, the initial vehicle speed $(v(0)=16 \mathrm{~km} / \mathrm{h})$ is greater than the reference speed $\left(v_{r e f}(0)=12 \mathrm{~km} / \mathrm{h}\right)$.

\section{Combined Longitudinal and Lateral Control}

In the previous sections, the lateral and longitudinal controllers were designed and validated through numerical simulations. In order to perform simultaneous path and speed tracking, NMPC for lateral control and the nonlinear longitudinal controller are combined in a global control architecture. This section details the interaction between these two controllers. 


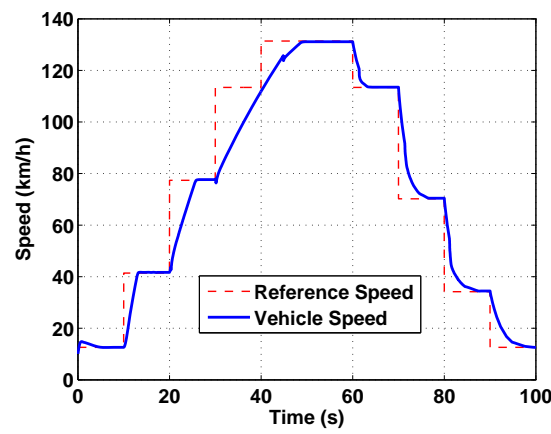

(a) Reference and vehicle speeds

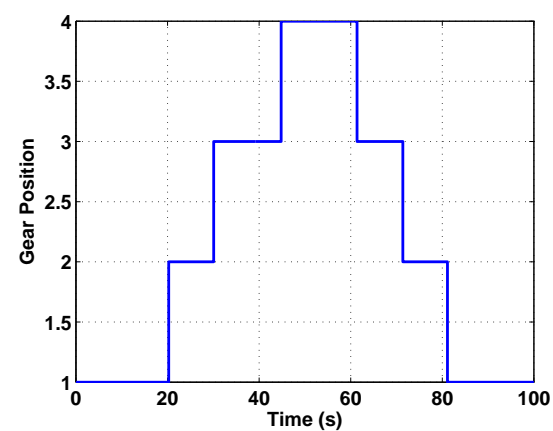

(c) Gear shifts

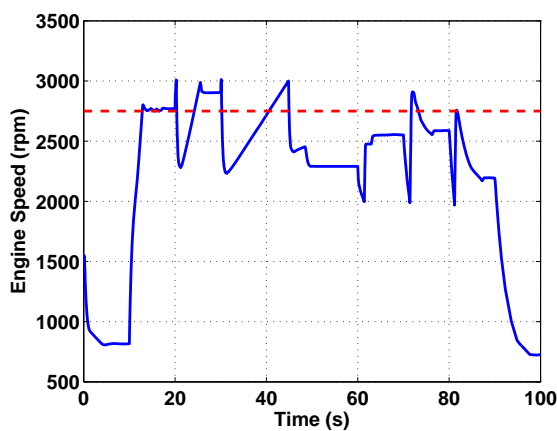

(b) Engine speed

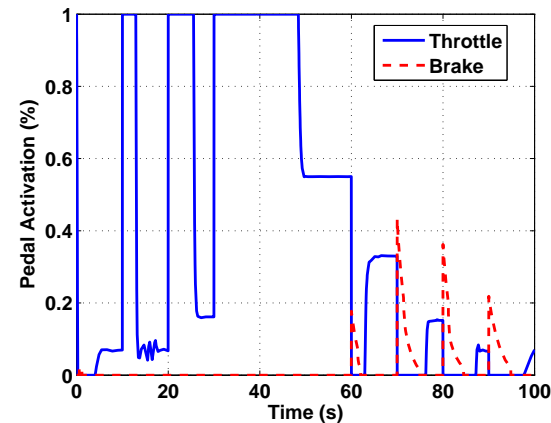

(d) Throttle and brake switching

Figure 6. Longitudinal speed tracking.

\subsection{Nonlinear Longitudinal and Lateral Control Design}

The aim of the global guidance architecture is to safely achieve autonomous driving. The control strategy proposed here is considered in the global guidance architecture depicted in Figure 7. The architecture can be decomposed into three levels called Perception, Reference Generation and Control:

- The Perception of the vehicle environment is of the utmost importance in the guidance architecture as it defines the environment in which the vehicle evolves. Its role is to provide the Reference Generation with the necessary information .

- The Reference Generation provides reference signals. It allows the calculation of the geometric trajectory which defines the path to be followed as well as the reference speed profile. These two different reference signals calculated at this level are used by Control.

- The Control ensures the automated vehicle guidance along the generated trajectories providing the appropriate control signals, here the throttle opening, the brake pressure and the steering angle. Simultaneous longitudinal and lateral control is necessary to guarantee efficient vehicle guidance.

The architecture shown in Figure 7 highlights the interaction between the lateral and longitudinal controllers. Indeed, the lateral control is designed following a path tracking approach which helps to decouple the speed tracking and the vehicle positioning problems. However, the coupling of the longitudinal and lateral dynamics is handled by the nonlinear prediction model. Following the MPC paradigm, it is assumed that the state-vector and the control input are available and the future evolution of the system is calculated using the prediction model. The prediction model used here has two control inputs, i.e. the steering angle and the torques on the wheels. The steering angle is the variable of interest for lateral control and 


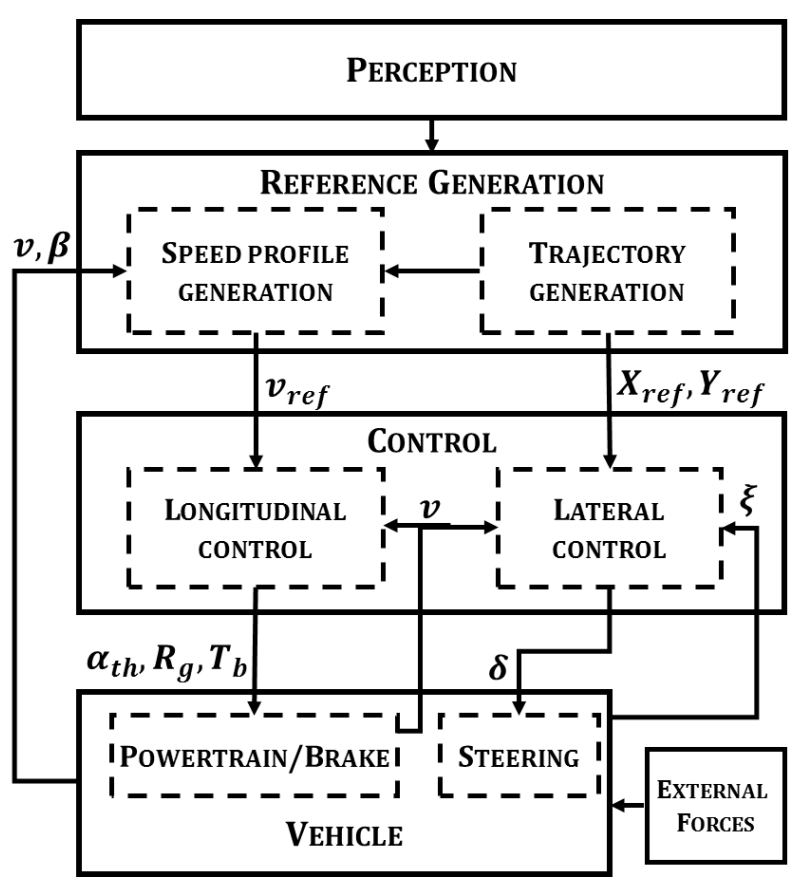

Figure 7. Architecture of the control strategy.

constitutes the optimization vector in the NMPC problem. The applied torques are supposed to be constant over the prediction horizon. Knowing that the prediction horizon does not exceed $100 \mathrm{~ms}$, this assumption is easily verified at least in non extreme driving situations. In this way, NMPC and the nonlinear longitudinal controller ensure the coupled path and speed tracking.

Note that no active lateral stabilization aspect is considered in the control design. In extreme lateral manoeuvres, vehicle stability may then be lost, e.g. when large steering manoeuvres are performed at high speed. In order to preserve vehicle lateral stability during guidance, the longitudinal reference speed should be adapted. To do so, a reference speed profile generator has been adopted.

\subsection{Reference Speed Profile Generator}

The role of the speed profile generator is to calculate the adequate longitudinal reference speed following a number of criteria. A first reference speed is calculated by the Reference Generation (see Figure 7) then, taking dynamics information into account, the speed profile generator adapts the reference speed profile according to the situation.

\subsubsection{Road information criteria}

The performance of lateral tracking largely depends on the vehicle longitudinal speed. The vehicle speed becomes critical when approaching a bend. Therefore, it should be calculated taking account of the road geometry information. As proposed in [9], the maximum longitudinal speed considering the road curvature is given by:

$$
v_{\max }=\sqrt{\frac{g \mu}{\rho_{r}}}
$$

where $g, \mu$ and $\rho_{r}$ are respectively the gravity, the friction coefficient and the road curvature. The description given by this criterion is incomplete and may be inappropriate to determine the maximum admissible speed. For this reason, more 
elaborate models and criteria are proposed. For the calculation of the maximum entry speed in bends, the National Highway Traffic Safety Administration (NHTSA) recommends the following model [24]:

$$
v_{\max }=\sqrt{\frac{g}{\rho_{r}}\left(\frac{\phi_{r}+\mu}{1-\phi_{r} \mu}\right)},
$$

where $\phi_{r}$ is the road camber angle. Then, the acceleration $a$ which should be applied to bring the speed of the vehicle to the maximum admissible speed given by (42) should be less than:

$$
a_{\max }=\sqrt{\frac{v^{2}-v_{\max }^{2}}{2\left(d-t_{r} v\right)}},
$$

where $v$ is the current vehicle speed, $d$ the distance to the summit of the bend and $t_{r}$ the time-delay due to the driver's reaction.

Criteria (41) and (42) only depend on the road geometry and can be evaluated in real time as far as the road information is available. In the proposed architecture (cf. Figure 7) the reference generation provides this information in real-time. Nevertheless, these criteria do not make use of the information on the lateral vehicle dynamics. Here, they are combined with the criteria on lateral vehicle stability presented below.

\subsubsection{Lateral stability criteria}

The information obtained from lateral dynamics are of the utmost importance to preserve vehicle lateral stability. One way to preserve lateral stability is to keep the vehicle far from critical situations. To this end, the vehicle speed in lateral manoeuvres is decisive. Note that the objective is the guidance of the vehicle in normal driving situations; active stabilization is not sought here. Different criteria on lateral stability are available in the literature, among them, the following criterion is of great interest [14]:

$$
\left|\frac{1}{24} \dot{\beta}+\frac{4}{24} \beta\right| \leq 1,
$$

where $\beta$ is the sideslip angle. Another criterion also using the side-slip angle $\beta$ and the vehicle speed $v$ is given by [30]:

$$
\beta \leq 10^{o}-7^{o} \frac{v^{2}}{(40 m / s)^{2}} .
$$

These criteria are investigated through simulations and roughly show the same stability threshold. However, the first criterion (44) needs the derivative of the side-slip angle and so it can be noise sensitive since the side-slip angle is calculated from measurements. Therefore, the second criterion (45) is preferred here.

\subsubsection{Reference speed calculation}

The Reference Generation provides the coordinates of the geometric trajectory to be followed by the vehicle and a speed profile taking legal speed limits into account. This profile is adapted considering the vehicle longitudinal dynamics limits and the criteria (41) and (45). The speed profile obtained is used as the reference signal by the longitudinal controller. 


\subsection{Tests and Simulations}

The performance of combined longitudinal and lateral control is investigated through simulations. As discussed above, the longitudinal controller is considered in the whole guidance strategy. Figure 8 shows the simulation results of automated guidance on the track shown in Figure 8(a) which corresponds to a highway exit. The trajectory reference is obtained from the Reference Generation module and the reference cruise speed is calculated as discussed in [1]. As can be seen in Figure $8(\mathrm{c})$, the lateral position error does not exceed $6 \mathrm{~cm}$. Figure $8(\mathrm{~d})$ shows that the longitudinal reference speed is also well tracked. Thanks to the proposed combined longitudinal and lateral control architecture, the whole guidance strategy provides good tracking performance.

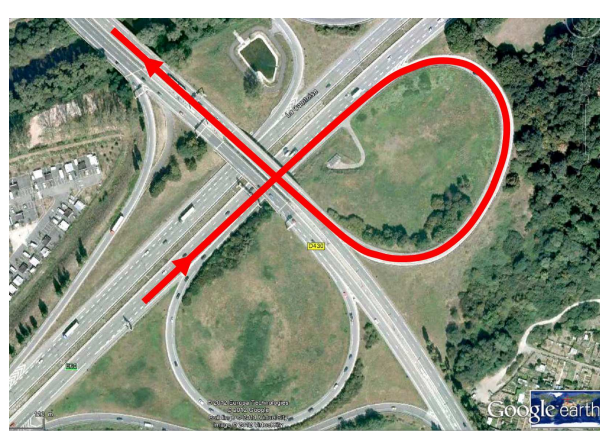

(a) Real-world road

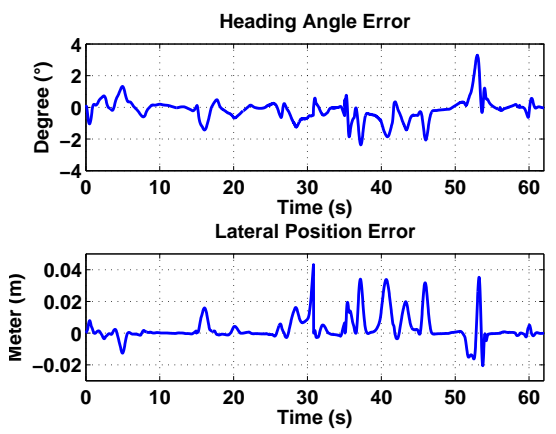

(c) Tracking errors

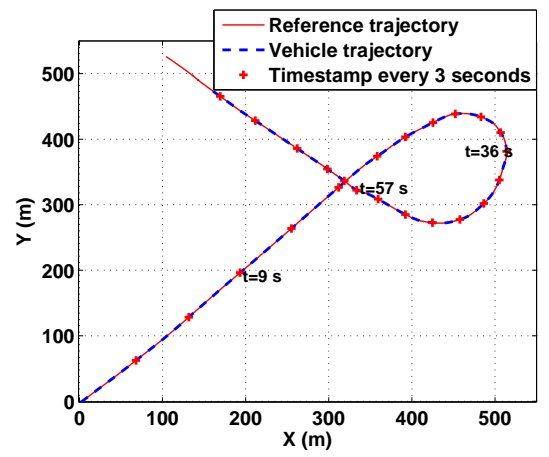

(b) Reference and vehicle trajectories

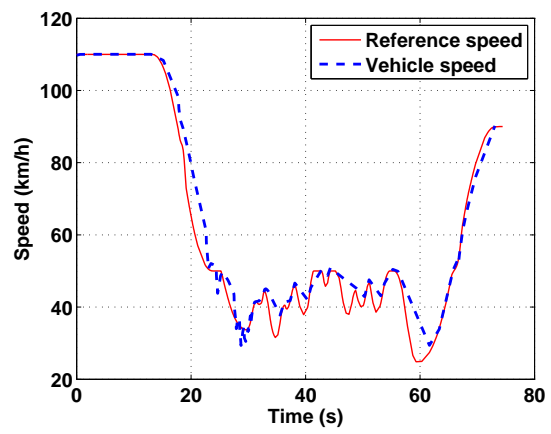

(d) Reference speed tracking

Figure 8. Combined longitudinal and lateral control test.

\section{Conclusion and Future Work}

This paper proposes a model-based control strategy for both longitudinal and lateral control. Lateral guidance is mainly ensured by a nonlinear model predictive controller. Longitudinal control is also based on a nonlinear control law considering the powertrain dynamics and gearbox ratio. Several simulations show the effectiveness of lateral control in performing path tracking at variable speeds. The simulations show highly interesting results for combined longitudinal and lateral control. The lateral position and heading angle errors are admissible and the longitudinal speed reference is correctly tracked. 
The results obtained in this work are promising and will be implemented on a test vehicle for experimental validation. The architecture proposed helps to consider new innovative aspects, not presented here, such as eco-friendly driving. The Reference Generation can be improved by taking account of the road slope in the trajectory generation.

\section{References}

[1] R. Attia, J. Daniel, J-P. Lauffenburger, R. Orjuela, and M. Basset. Reference Generation and Control Strategy for Automated Vehicle Guidance. In IEEE Intelligent Vehicles Symposium (IV'12), Alcalá de Henares, Spain, 2012.

[2] R. Attia, R. Orjuela, and M. Basset. Coupled Longitudinal and Lateral Control Strategy Improving Lateral Stability for Autonomous Vehicle. In American Control Conference (ACC'12), Montreal, Canada, 2012.

[3] T. Besselmann. Constrained Optimal Control of Piecewise Affine and Linear Parameter-Varying Systems. PhD thesis, ETH Zürich, 2010.

[4] S. Di Cairano, H.E. Tseng, D. Bernardini, and A. Bemporad. Steering Vehicle Control by Switched Model Predictive Control. In 6th IFAC Symposium Advances in Automotive Control, Munich, Germany, 2010.

[5] J. Daniel. Trajectory Generation and Data Fusion for Control Oriented Advanced Driver Assisstance Systems. PhD thesis, Université de Haute-Alsace, 2010.

[6] K. ElMajdoub, F. Giri, H. Ouadi, L. Dugard, and F.Z. Chaoui. Vehicle Longitudinal Modeling for Nonlinear Control. Control Engineering Practice, 20:69-81, 2012.

[7] P. Falcone, F. Borrelli, J. Asgari, H. E. Tseng, and D. Hrovat. Predictive Active Steering Control for Autonomous Vehicle Systems. IEEE Transaction on Control Systems Technology, 15 No. 3:566-580, 2007.

[8] A. Ferrara and C. Vecchio. Second Order Sliding Mode Control of Vehicles with Distributed Collision Avoidance Capabilities. Mechatronics, 19:471-477, 2009.

[9] A. Gallet, M. Spigai, and M. Hamidi. Use of Vehicle Navigation in Driver Assistance Systems. In IEEE Intelligent Vehicles Symposium (IV'00), Derborn, MI, USA, 2000.

[10] L. Grüne and J. Pannek. Nonlinear Model Predictive Control. Springer, 2011.

[11] L. Guzzella. Automobiles of the Future and the Role of Automatic Control in those Systems. Annual Reviews in Control, 33:1-10, 2009.

[12] L. Guzzella and C. H. Onder. Introduction to Modeling and Control of Internal Combustion Engine Systems. Springer-Verlag, 2004.

[13] L. Guzzella and A. Sciarretta. Vehicle Propulsion Systems. Springer-Verlag, 2005.

[14] J. He, D. A. Crolla, M. C. Levesley, and W. J. Manning. Coordination of Active Steering, Driveline, and Braking for Integrated Vehicle Dynamics Control. J. Automobile Engineering, 220 Part D:1401$1421,2006$.

[15] U. Kiencke and C. Nielsen. Automotive Control Systems. Springer-Verlag, 2000.

[16] S-J Kwon, T. Fujioka, K-Y Cho, and M-W Suh. Model-matching Control Applied to Longitudinal and Lateral Automated Driving. J. Automobile Engineering, 219 Part D:583-598, 2005.

[17] E. M. Lim. Lateral and Longitudinal Vehicle Control Coupling in the Automated Highway System. Master's thesis, University of California at Berkeley, 1998.

[18] H-T. Luu, L. Nouvelière, and S. Mammar. Dynamic Programming for Fuel Consumption Optimization on Light Vehicle. In 6th IFAC Symposium Advances in Automotive Control (AAC'10), Munich, Germany, 2010.

[19] E. Maalouf, M. Saad, and H. Saliah. A Higher Level Path Tracking Controller for a Four-Wheel Differentially Steered Mobile Robot. Robotics and Autonomous Systems, 54:23-33, 2006.

[20] L. Menhour, B. d'Andréa Novel, C. Boussard, M. Fliess, and H. Mounier. Algebraic Nonlinear Estimation and Flatness-based Lateral/Longitudinal Control for Automotive Vehicles. In International Conference on Intelligent Transportation Systems (ITSC'11), Washington, DC, USA, 2011.

[21] L. Nehaoua and L. Nouvelière. Backstepping Based Approach for the Combined Longitudinal-Lateral Vehicle Control. In IEEE Intelligent Vehicle Symposium (IV'12), Alcalá de Henares, Spain, 2012.

[22] L. Nouvelière and S. Mammar. Experimental Vehicle Longitudinal Control Using a Second Order Sliding Mode Technique. Control Engineering Practice, 15:943-954, 2007.

[23] E. Onieva, J.E.Naranjo, V. Milanés, J. Alonso, R. Garcia, and J. Pérez. Automatic Lateral Control for Unmanned Vehicles via Genetic Algorithms. Applied Soft Computing, 11:1303-1309, 2010.

[24] D. Pomerleau, T. Jochem, C. Thorpe, P. Batavia, D. Pape, J. Hadden, N. McMillan, N. Brown, and J. Everson. Run-off-road Collision Avoidance Using IVHS Countermeasures. Technical report, Department of Transportation, NHTSA, 1999.

[25] Rajesh Rajamani. Vehicle Dynamics and Control. Springer, 2006.

[26] P. Shakouri, A. Ordys, M. Askari, and D. S. Laila. Longitudinal Vehicle Dynamics Using Matlab/Simulink. In UKACC International Conference on Control, Coventry, UK, 2010.

[27] Y. Shibahata. Progress and Future Direction of Chassis Control Technology. Annual Reviews in Control, 29:151-158, 2005.

[28] J.M. Snider. Automatic Steering Methods for Autonomous Automobile Path Tracking. Technical report, Robotics Institute, Carnegie Mellon University, Pittsburgh, Pensylvania, 2009.

[29] S. Thrun, David Stavens Andrei Aron James Diebel Philip Fong John Gale Morgan Halpenny Gabriel Hoffmann Kenny Lau Celia Oakley Mark Palatucci Vaughan Pratt Pascal Stang Sven Strohband Cedric Dupont Lars-Erik Jendrossek Christian Koelen Charles Markey Carlo Rummel Joe van Niekerk 
Eric Jensen Philippe Alessandrini Gary Bradski Bob Davies Scott Ettinger Adrian Kaehler Ara Nefian Mike Montemerlo, Hendrik Dahlkamp, and Pamela Mahoney. Stanley: The Robot that Won the DARPA Grand Challenge. journal of Field Robotics, 23:661-692, 2006.

[30] P. Tondel and T. A. Johansen. Control Allocation for Yaw Stabilization in Automotive Vehicles using Multiparametric Nonlinear Programming. In American Control Conference (ACC'05), Portland, USA, 2005.

[31] L. Wang. Model Predictive Control System Design and Implementation Using MATLAB. SpringerVerlag, 2009.

[32] L. Xiao and F. Gao. A Comprehensive Review of the Development of Adaptive Cruise Control Systems. Vehicle System Dynamics: International Journal of Vehicle Mechanics and Mobility, 48(10):1167-1192, 2010.

[33] J. Yoon, W. Cho, J. Kang, B. Koo, and K. Yi. Design and Evaluation of a Unified Chassis Control System for Rollover Prevention and Vehicle Stability Improvment on a Virtual Test Track. Control Engineering Practice, 18:585-597, 2010. 